\title{
Evolution of Two-Dimensional Soap-Film Networks
}

\author{
C. W. J. Beenakker \\ Philips Research Laboratories, 5600 JA Eindhoven, The Netherlands \\ (Received 14 July 1986)
}

\begin{abstract}
A mean-field theory is presented for the coarsening of a two-dimensional soap-film network. This theory explains (1) the correlation between area and number of sides of the cells, and (2) the anomalous absence of dynamical scaling laws, which is shown to have its origin in the topological constraint of a space-filling network.
\end{abstract}

PACS numbers: $82.70 . \mathrm{Rr}, 68.90,+\mathrm{g}$

Coarsening of soap froths is an interesting and familiar process in which soap cells lower their surface free energy by increasing their average size. Traditionally, metallurgists have studied this process as a model for grain growth in polycrystalline solids. ${ }^{1}$ Recently, cellular soap-film networks have appeared in the literature on condensed matter as prototypical systems with topological disorder. ${ }^{2}$ It is, in fact, the topological aspect that distinguishes this process from what is known as Ostwald ripening, which is the coarsening of precipitated grains in a solution. Whereas there one has well-separated, approximately spherical grains, here the cell shapes are constrained by the network topology: Spherical cells cannot fill space. For Ostwald ripening, a very successful mean-field theory was developed around 1960 by Lifshitz, Slyosov, and Wagner ${ }^{3,4}$ (LSW). To my knowledge, there is no comparable theory that incorporates the topological constraints of a network. It is the purpose of this paper to present such a mean-field theory for the coarsening of a two-dimensional soap-film network. Experiments ${ }^{5,6}$ have indicated that this system does not follow the LSW scaling laws, and one aim of the theory is to explain this anomalous behavior. A second aim is to explain the relation between shape and size of cells in the network.

The structure of a soap froth between two closely spaced parallel plates can be described by a twodimensional network with threefold coordination of the vertices (see Fig. 1). Pressure differences of the gas in adjacent cells drive the gas through the cell walls. The time scale of this diffusion process is observed to be much larger than the time scale on which the soap film adjusts its shape to variations in pressure, so that one may assume that the soap-film network is continuously in equilibrium with the gas in each cell. ${ }^{7}$ This implies, in particular, that cell walls are circular arcs meeting at angles of $120^{\circ}$. The pressure difference $\Delta p$ is given by Laplace's law, $\Delta p=\sigma / R$, where $\sigma$ is the surface tension coefficient and $R$ the radius of curvature of the common boundary. It is assumed that the gas is incompressible and that its flux through the soap film equals $\mu \Delta p$, where $\mu$ is a permeability coefficient. For the growth rate of the area $A$ of an $n$-sided cell, one then easily derives von Neumann's law, 8,9

$$
d A / d t=k(n-6),
$$

with $k=(\pi / 3) \sigma \mu$. The total area of the system remains constant in time, as it should, by virtue of Euler's theorem that the cells have six sides on average. The description of the network dynamics consists of two problems, which can be dealt with separately as a result of the separation of time scales mentioned above: (1) What is the relation between the area and number of sides of the cells; and (2) how does the distribution of areas evolve in time? I first turn to problem (1).

It is observed experimentally that large cells tend to have many sides. I attribute this correlation to the relatively low surface energy of a many-sided cell. ${ }^{10}$ Consider an $n$-sided cell bounded by a regular polygon constructed by circular arcs meeting at $120^{\circ}$ angles. For a given length $S$ of the cell perimeter, its area $A$ is given by

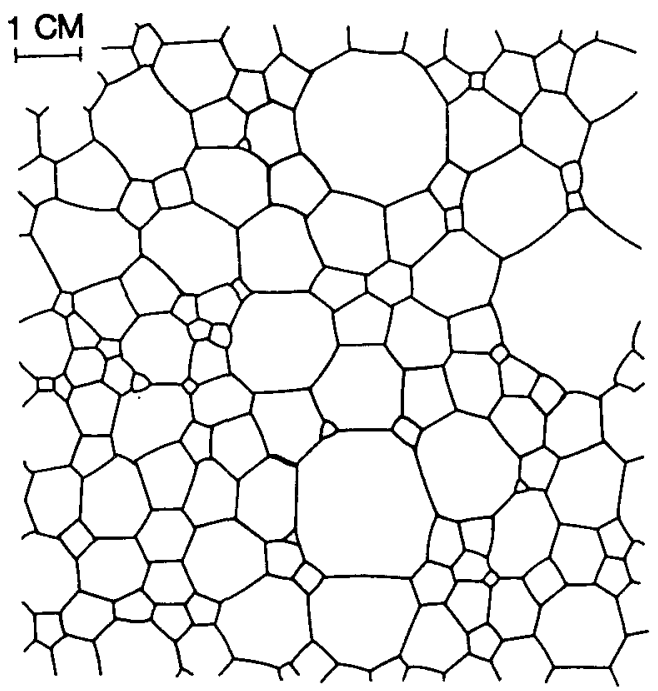

FIG. 1. Two-dimensional soap-film network, traced from an experimental photograph made by Smith (Ref. 5). The froth lies between parallel glass plates, spaced about $4 \mathrm{~mm}$ apart. 
$A=S^{2} \beta(n)$, with

$$
\beta(n)=(4 n)^{-1}(\pi / 6-\pi / n)^{-2} \sin ^{2}(\pi / 6-\pi / n)[\cot (\pi / n)+\cot (\pi / 6-\pi / n)]-(4 n)^{-1}(\pi / 6-\pi / n)^{-1} .
$$

The form function $\beta(n)$ increases monotonically by $4 \%$ as $n$ goes from 2 to $\infty$. Let me now express the free energy $F$ of the network solely in terms of the joint distribution $P(A, n)$ of area and number of sides. By the ignoring of higher order distribution functions, the effects of specific correlations between the sizes and shapes of neighboring cells are neglected in this "mean-field" theory. Consistent with this approximation I take the regular polygon as the representative cell shape, since deviations from regularity would result from local correlations which are neglected anyway. The free energy is then given by

$$
F=\int_{0}^{\infty} d A \sum_{n=2}^{\infty}\left\{\sigma[A / \beta(n)]^{1 / 2} P(A, n)+k_{\mathrm{B}} T P(A, n) \ln P(A, n)\right\} .
$$

To obtain the equilibrium state of the network this expression is minimized, given the distribution of areas $P(A)=\sum_{n=2}^{\infty} P(A, n)$, and subject to the topological constraint (Euler's theorem)

$$
\int_{0}^{\infty} d A \sum_{n=2}^{\infty}(n-6) P(A, n)=0
$$

Because of the fact that the surface energy of a soap cell is very much larger than the thermal energy $k_{\mathrm{B}} T$, it follows from this minimization problem that the distribution of the number of sides for a given area is nonvanishing for one integer only, i.e.,

$$
P(A, n)=P(A) \delta_{n, n_{c}\left(A / A_{c}\right)},
$$

with $n_{c}$ that integer $\geq 2$ for which the expression

$$
[A / \beta(n)]^{1 / 2}+3^{-13 / 4} 2^{-1 / 2}(5 \pi-9 \sqrt{3}) A_{c}^{1 / 2} n
$$

has the smallest value. ${ }^{11}$ The characteristic area $A_{c}$ is a Lagrange multiplier, to be determined from the require-

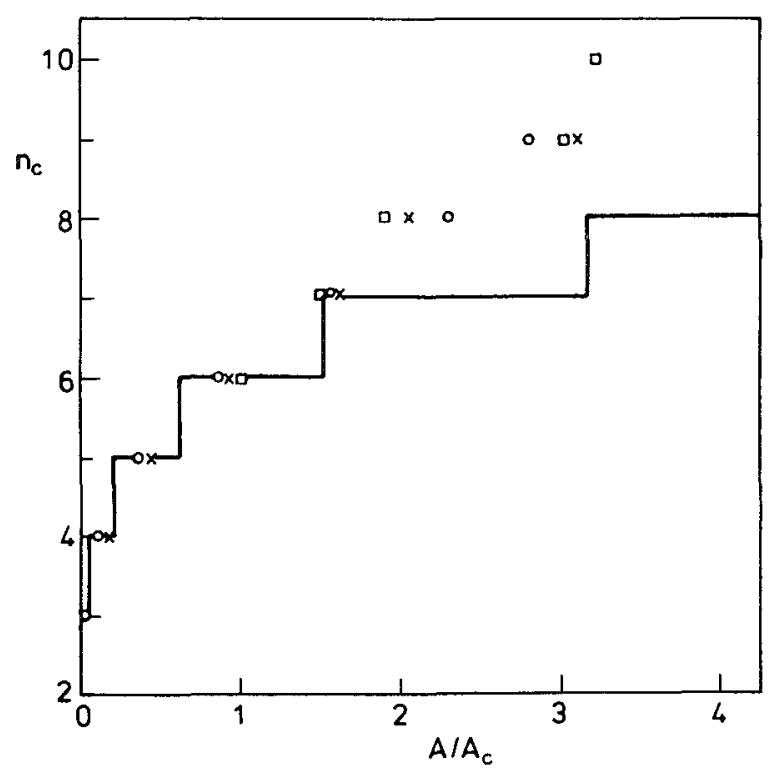

FIG. 2. Plot of the relation between cell area and number of sides, from the minimizing of expression (6). For comparison, markers show $\bar{A}(n) / \bar{A}$ as obtained in the simulation of Ref. 12 . (The different markers correspond to three different networks; for $n \leq 5$, squares and circles coincide.) ment that $P(A, n)$ satisfies Eq. (4). In general $A_{c}$ is somewhat smaller than, but of the order of, the average area $\bar{A}$. The one-to-one relation between area and number of sides which follows from Eqs. (5) and (6) is plotted in Fig. 2, together with numerical data for the average area $\bar{A}(n)$ of an $n$-sided cell obtained by Weaire and Kermode, ${ }^{12}$ from simulating the equilibration of networks of 100 cells. (No quantitative experimental data seem to be available.) The agreement is quite satisfactory, in view of the simplicity of the theory. Note, in particular, that both sets of data show a sublinear correlation, unlike the linear correlation observed in botanical cellular networks (Lewis's law; see Ref. 2), although the numerical data increase more rapidly for large areas than predicted by the mean-field theory. It would be important to have also numerical (or experimental) data for the average number of sides of cells with a given area, in order to see to what extent correlation effects smooth out the steps in Fig. 2.

I now continue with problem (2), the time dependence of the area distribution function $P(A, t)$. Combining the result (5) with von Neumann's law (1), one obtains the evolution equation

$$
\begin{aligned}
& \partial P(A, t) / \partial t \\
& \quad=-k(\partial / \partial A)\left\{P(A, t)\left[n_{c}\left(A / A_{c}(t)\right)-6\right]\right\},
\end{aligned}
$$

where the growth rate $n_{c}\left(A / A_{c}\right)-6$ has been determined above (see Fig. 2), and $A_{c}$ is such that $\bar{n}_{c}\left(A / A_{c}\right)=6$. This equation looks very similar to the one presented in the LSW theory of Ostwald ripening. ${ }^{3,4}$ The celebrated result of that theory is that, in the long-time limit, the (normalized) distribution function depends only on $A$ and $t$ through the scaled variable $A / \bar{A}(t)$, with $\bar{A}(t)$ increasing linearly with time. Surprisingly enough, no such scaling law has been observed in coarsening soap-film networks. ${ }^{5,6}$ Instead, available experimental data show a distribution which broadens without any indication of an asymptotic limit. Though apparently similar to the LSW equation, Eq. (7) nonetheless exhibits such anomalous behavior. This is shown in Fig. 3, obtained by a numerical integration. ${ }^{13}$ I started with a network consisting of hexagonal cells with a small fraction of pentagon-heptagon pairs. The hexagonal network is unstable against 


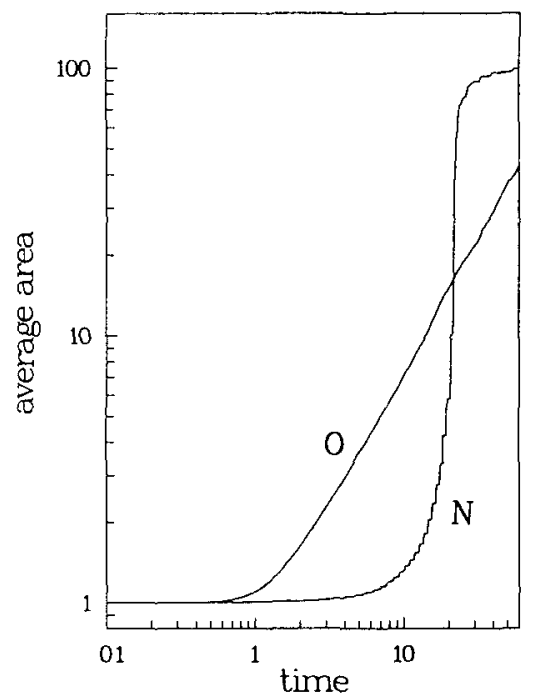

(a)

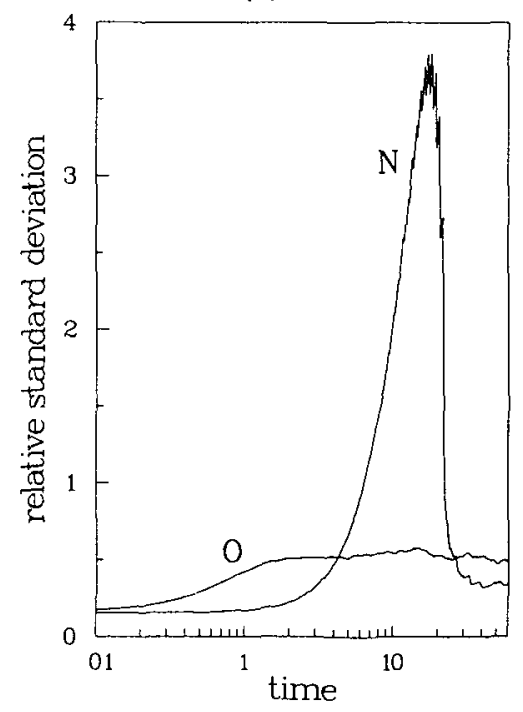

(b)

FIG. 3. Time dependence, from Eq. (7), of the average cell area $\bar{A}$ and of the relative standard deviation $\left\langle(A / \bar{A}-1)^{2}\right\rangle^{1 / 2}$ in the network, indicated by curve $N$. Curve $O$ results from replacing the step function $n_{c}\left(A / A_{c}\right)$ in the growth rate by the smooth function $2+4\left(A / A_{c}\right)^{1 / 3}$, and shows the scaling regime found in Ostwald ripening. [Units: Area in units of the initial average area $\vec{A}(0)$; time in units of the coarsening time scale $\bar{A}(0) / k$, see Ref. 8 .]

the formation of such "5-7 defects," new defects are created, and the distribution broadens. The distribution does not then approach a scale-invariant form, but continues to broaden. Finally, a "collapse" occurs, which consists of the rapid, almost total disappearance of fewsided cells and which returns the system to a state with mostly hexagons plus a few defects. The process then repeats itself. Shortly before the collapse only a small fraction of hexagonal cells remains and the distribution of the number of sides has become bimodal, with a standard de- viation around 3.2. I have no analytic derivation of a final collapse of the distribution function, but it does not seem to be an artifact of the finite number of cells employed in the integration routine (see Ref. 13)-which might be a source of concern with such a broad distribution. In any case it should be realized that the point at which the collapse observed here occurs lies outside the range of samples analyzed by Aboav ${ }^{5}$ (which has a maximum standard deviation of 1.7 in the distribution of the number of sides), and this would explain why it has not been seen in those experiments.

What is the origin of the anomalous coarsening of the network? By application of the Lifshitz-Slyosov method of analysis ${ }^{3,14}$ to Eq. (7), the absence of scaling laws can be traced to the plateaus in the growth rate (see Fig. 2), and thus ultimately to the topological constraint of a space-filling network. This is illustrated in Fig. 3, where it is shown that the LSW scaling regime of grain growth (Ostwald ripening) is recovered if the step function of Fig. 2 is replaced by a smooth curve. It is remarkable how drastically the network topology affects its dynamics. The evolution of the network (curve $N$ in Fig. 3) differs from the Ostwald ripening dynamics (curve $O$ ) in two respects: (1) Instead of the linear time dependence of the average area, corresponding to a constant coarsening rate, the network shows two alternating regimes of slow and fast growth. The effective coarsening rate of the network, averaged in time over the two regimes, is roughly the same as in the Ostwald ripening process. (2) Instead of reaching a time-independent scaled distribution of areas, the network shows a scaled distribution which, in the above two regimes, alternately broadens and collapses. Note that the time scale of this alternation is in general very slow: Use of the numerical values from Ref. 8 gives a duration of $10 \mathrm{~d}$ for the initial regime of a broadening distribution. Aboav's data, ${ }^{5}$ in contrast, extends only over a period of about $4 \mathrm{~d}$, and is indeed still well in the broadening regime. Clearly, more extensive measurements are needed.

In summary, the theory presented above, based on a simple "mean-field" free energy, offers an explanation for the intriguing nonscaling dynamics of $2 \mathrm{D}$ soap-film networks. It is shown for the first time that such anomalous behavior can be obtained by incorporation of topological constraints into the classical LSW theory of grain growth. More experimental data are needed to determine the importance of neglected effects, in particular of correlations.

Valuable discussions with Professor P. Mazur, Professor P. Nozières, and Professor M. F. H. Schuurmans are gratefully acknowledged.

Note added.-In a recent publication, Wejchert, Weaire, and Kermode ${ }^{15}$ report on the numerical simulation of a soap-froth model which is similar to (but not identical with) the model analyzed above. They do not find the anomalous behavior seen in the experiments 
analyzed in Refs. 5 and 6, and stress the need for more experimental measurements.

${ }^{1}$ C. S. Smith, in Metal Interfaces, edited by C. Herring (American Society for Metals, Cleveland, 1952).

${ }^{2} \mathrm{D}$. Weaire, in Topological Disorder in Condensed Matter, edited by F. Yonezawa and T. Ninomiya (Springer, Berlin, 1983).

3I. M. Lifshitz and V. V. Slyosov, J. Phys. Chem. Solids 19, 35 (1961). See also E. M. Lifshitz and L. P. Pitaevskii, Physical Kinetics (Pergamon, Oxford, 1981), paragraph 100.

${ }^{4}$ C. Wagner, Z. Elektrochem. 65, 581 (1961).

${ }^{5}$ D. A. Aboav, Metallography 13, 43 (1980).

${ }^{6}$ D. Weaire and J. P. Kermode, Philos. Mag. B 47, L29 (1983).

${ }^{7}$ Such a separation of time scales does not hold for grain growth in polycrystalline solids, and this is one point where its analogy to the coarsening of soap froths breaks down.

${ }^{8} \mathrm{~J}$. von Neumann, in Ref. 1, p. 108. Typically, $k \sim 10^{-7} \mathrm{~cm}^{2}$ $\mathrm{s}^{-1}$ at atmospheric pressure [see A. J. de Vries, Rec. Trav. Chim. 77, 209,283 (1958)]. The resulting coarsening time scale $\bar{A}(0) / k$ for an initial average cell area $\bar{A}(0) \sim 1 \mathrm{~mm}^{2}$ is of the order of $1 \mathrm{~d}$.

${ }^{9}$ It is assumed that diffusion of gas through cell walls is the dominant mechanism for changes in cell areas, and that coalescence of adjacent cells by any other mechanism may be neglected. Possible variations in time of the material parameters $\sigma$ and $\mu$ are also neglected.

${ }^{10} \mathrm{~A}$ different point of view has been taken by N. Rivier, Philos. Mag. B 52, 795 (1985). Rivier does not account for the surface-energy differences of cells of different shapes but equal areas, but considers only the entropy differences of cell configurations. Since the surface-energy differences involved are, however, very much larger than the thermal energy $k_{\mathrm{B}} T$, that approach does not seem justified.

${ }^{11}$ The numerical coefficient in expression (6) equals $\frac{1}{2} \beta^{\prime}(6) \beta(6)^{-3 / 2}$ [with $\beta^{\prime}(x)=d \beta(x) / d x$ ], so that $n_{c}=6$ when $A=A_{c}$.

${ }^{12}$ D. Weaire and J. P. Kermode, Philos. Mag. B 50, 379 (1984). I was not able to calculate the characteristic area $A_{c}$ of the networks generated in this paper, because of insufficient information on the area distributions. For the comparison in Fig. 2, I therefore approximated $A_{c}$ by the average area $\bar{A}$.

${ }^{13}$ I used a Monte Carlo technique of integrating a set of $N$ ordinary differential equations. $d A_{j} / d t=k\left[n_{c}\left(A_{j} / A_{c}\right)-6\right]$, $j=1,2, \ldots, N, t_{1}<t<t_{f}$, with $A_{j}\left(t_{1}\right)$ sampled from an initial distribution $P\left(A, t_{t}\right)$. I found this method quite robust, runs with $N=10^{3}$ and $N=10^{4}$ giving essentially the same results.

${ }^{14}$ The Lifshitz-Slyosov argument for dynamical scaling laws requires a growth rate $v\left(A / a_{c}\right)$ such that the function $\gamma_{v}(x)-x$ has a smooth maximum of zero for one particular value of the parameter $\gamma>0$. The growth rate $v(x)$ $=n_{c}(x)-6$ does not satisfy this requirement, since the maximum is necessarily reached right at a plateau edge of $n_{c}$.

${ }^{15} \mathrm{~J}$. Wejchert, D. Weaire, and J. P. Kermode, Philos. Mag. B 53,15 (1986). 